\title{
Politique
}

Politique

\section{Gérard Bergeron, À nous autres - aide-mémoire politique par Ie temps qui court, Montréal, Québec-Amérique, 1986, 210 p.}

\section{François Rocher}

Numéro 11, hiver 1987

L’État privé

URI : https://id.erudit.org/iderudit/040554ar

DOI : https://doi.org/10.7202/040554ar

Aller au sommaire du numéro

Éditeur(s)

Société québécoise de science politique

ISSN

0711-608X (imprimé)

1918-6584 (numérique)

Découvrir la revue

Citer ce compte rendu

Rocher, F. (1987). Compte rendu de [Gérard Bergeron, À nous autres -

aide-mémoire politique par Ie temps qui court, Montréal, Québec-Amérique,

1986, 210 p.] Politique, (11), 167-171. https://doi.org/10.7202/040554ar d'utilisation que vous pouvez consulter en ligne.

https://apropos.erudit.org/fr/usagers/politique-dutilisation/ 
Gérard Bergeron, À nous autres - aide-mémoire politique par le temps qui court, Montréal, Québec-Amérique, 1986, 210 p.

Peu d'ouvrages d'analyse politique ont proposé une relecture détaillée de la période post-référendaire. C'est à cette tâche que s'est consacré Gérard Bergeron dans son dernier volume. Cette contribution vise globalement deux objectifs: d'abord prendre la mesure de l'échec référendaire et d'en identifier les principales conséquences non seulement sur le plan constitutionnel, ce qui fut largement accompli par d'autres observateurs, mais plus largement sur l'avenir collectif de la société québécoise; ensuite proposer une mise en situation des principaux problèmes qui confrontent la situation politique actuelle du Québec. L'intention première de l'auteur est clairement présentée dès l'introduction, «ce texte est un effort personnel de mise au point pour d'abord m'y retrouver» (p. 12) et, un peu plus loin de nous dire que «je souhaiterais surtout que ces lignes rejoignent tous ceux qui éprouvent, comme l'auteur, le besoin d'établir nos coordonnées politiques actuelles le moins mal possible» (p. 13). En ce sens, la facture générale de l'ouvrage emprunte davantage à l'essai qu'à toute autre forme d'écriture. Pour les lecteurs familiers de Bergeron, cet ouvrage se veut une poursuite de la réflexion entreprise dans son analyse de l'expérience du Parti québécois dans «Pratique de l'État au Québec publiée en 1984 ainsi que de son étude de personnalités dans Notre miroir à deux faces: Trudeau - Lévesque, paru en 1985.

La première partie de l'écrit est consacrée à l'analyse de l'échec référendaire mais surtout aux nombreux problèmes rencontrés par le gouvernement du Parti québécois au cours de la période 1980-1985. Ce dernier a dû affronter un nombre impressionnant de problèmes que Bergeron transpose en empruntant de façon imaginative et intéressante la symbolique biblique des dix plaies d'Égypte. 
La première "plaie», l'eau changée en sang, correspond dans l'imaginaire de Bergeron à l'échec référendaire, suivie d'une seconde, tout aussi dévastatrice associée au ravage des grenouilles, que fut la contre-offensive constitutionnelle menée par Trudeau. Outre ces deux facteurs de crise, plusieurs autres peuvent être associés directement ou indirectement au NON référendaire: l'isolement du Québec après les disgracieuses tractations de la conférence constitutionnelle de novembre 1981, les déchirements du Parti québécois et les manœuvres de récupération de Lévesque, la mise au rancart de l'option souverainiste. Cependant, toutes ces "plaies» ne sont pas le seul résultat de ce fameux 20 mai 1980 puisqu'il faut aussi prendre en compte la crise économique, la démobilisation partisane et l'affrontement entre l'État et ses employés. Cette kyrielle d'épreuves pavait le chemin à la dixième et ultime "plaie» correspondant au retour du Parti québécois sur les bancs de l'opposition.

Cette réécriture québécoise du récit du livre de l'Exode nous permet de resituer certains grands moments qui ont marqué la conjoncture politique des six dernières années. Toutefois, hormis la fraîcheur du récit et le ton incisif, le lecteur qui aura suivi un tant soit peu l'actualité n'apprendra rien de neuf à la lecture de ces pages. L'intérêt de cette première partie réside surtout dans la réflexion qu'en tire Bergeron, notamment en ce qui concerne les conséquences de l'échec référendaire. Pour ce dernier, «même les épreuves qui n'y avaient pas de rapport direct feront d'autant plus mal au Parti québécois qu'il n'a pas réussi, dans la suite, à compenser par des succès sur d'autres plans cette sérieuse déperdition d'énergie politique» (p. 62.). La déception politique sera d'autant plus grande que l'espoir d'une réforme allant dans le sens d'un fédéralisme plus avantageux pour le Québec n’a pas été comblé. Élargissant son propos, l'auteur nous rappelle sa position selon laquelle le gouvernement du PQ ne pouvait gagner la consultation référendaire, bien que l'expérience valait d'être tentée: 
«il y avait assez longtemps que la symbolique indépendantiste traînait dans les coulisses de la conscience nationale sujette à l'assoupissement» (p. 79). Cet échec était prévisible pour plusieurs raisons. D'abord, le projet de souveraineté-association, du fait de son ambiguité, s'est accompagné d'une stratégie fluctuante et imprécise dont une des faiblesses, et non la moindre, résidait dans la conviction que l'autre partie (les autres parties, compte tenu de la forme fédérative de l'État canadien), dont on espérait la défaite, prendrait volontairement part à de futures négociations. Parmi la multitude des considérations évoquées par Bergeron, la plus importante semble être la stratégie employée par le PQ au cours des années 1970, à travers l'étapisme et le bon gouvernement, stratégie qui allait à l'encontre de son objectif de réaliser la souveraineté-association. Pour un parti souverainiste, tenir le pari de la gouverne dans un système par ailleurs décrié discréditait ses propres arguments. Selon Bergeron, "gagner sans livrer bataille décisive...; sortir du système, sans le vaincre ni pouvoir le détruire, tout en continuant d'y fonctionner et d'en attendre les avantages... C'était trop espérer de la providence des peuples en mal d'indépendance nationale» (p. 67). L'indépendance est un fait de nature révolutionnaire, nous rappelle Bergeron, parce qu'il s'agit d'un phénomène anti-système. Or, pour réussir, le projet péquiste aurait eu besoin d'être accompagné par un dysfonctionnement de la fédération comme système: "bref, il eût fallu une situation irréelle pour que la velléité centrifuge du gouvernement d'un État fédéré ait eu bien davantage d'efficacité» (p. 68).

La seconde partie de l'ouvrage nous rappelle les récentes successions dans les leaderships des partis et les remplacements de gouvernement, tant au niveau fédéral que provincial. S'accrochant aux développements de l'actualité, l'auteur fait état de la prise de pouvoir du gouvernement Mulroney et des difficultés rencontrées. Moins sous forme de chronique, Bergeron aborde par la suite le «mystère de la résurrection politique de Robert Bourassa», sou- 
lignant une constante du Parti libéral du Québec qui est son opportunisme et sa capacité de se faire fort des faiblesses du camp adverse pour finir par le déloger, d'autant plus qu'il n'a pas à faire face à de contestation véritable comme parti, se la réservant comme gouvernement. Cette partie souligne les enjeux des élections de décembre 1985 pour finalement évaluer les conséquences de l'isolement constitutionnel du Québec.

La dernière partie de l'essai va au-delà de la simple mesure de la morosité politique qui semble affliger le Québec pour considérer certains problèmes fondamentaux (une dizaine) qui confrontent actuellement le Québec dont les questions démographique et linguistique. L'intérêt de l'exercice ne réside pas tant dans la nouveauté des aspects considérés, qui prennent souvent l'allure de descriptions, que dans leur réunion sous une même problématique, celle d'une interrogation sur le devenir collectif. À ce sujet, Bergeron ne sombre ni dans le défaitisme d'un inéluctable affaiblissement de la société québécoise ni dans le triomphalisme volontariste qui a tant marqué les derniers vingt-cinq ans. Il parle du Québec comme d'une société politiquement "amortie» mais encore vivace. La défaite du Parti québécois, et la trève du discours sur l'indépendance, n’a pas marqué la fin du nationalisme québécois mais indique un sévère déclin des circonstances. Selon Bergeron, "une situation cumulative d'insatisfactions économiques serait encore susceptible de ranimer, un de ces jours, la fibre politique engourdie du nationalisme Québécois. Mais, à aucun signe actuellement visible, la réactivation de ce nationalisme n'ira jusqu'à l'objectif d'une indépendance nationale» (p. 174). Si l'ouvrage de Bergeron ne se termine pas sur un grand Projet ni sur une programmatique, les questions et les défis soulevés ne sont toutefois pas sans pertinence.

Gérard Bergeron nous propose donc, à travers cet essai, une relecture intéressante des événements qui ont marqué l'actualité politique de la première moitié de la décennie. Incidemment, le 
sous-titre d'aide-mémoire politique par les temps qui court n'aurait pu être mieux choisi pour décrire l'ouvrage. Mais l'intérêt ne s'arrête pas à ce rappel de notre récent passé. Il se fonde sur le traitement personnel qui en est fait qui donne une nouvelle coloration à des événements qui apparaissent à première vue bien ternes.

François Rocher

Université de Montréal 